\title{
Réponse à François-René Picon, « Ce qu'esclave veut dire »
}

Alain Testart

\section{(2) OpenEdition}

1 Journals

Édition électronique

URL : https://journals.openedition.org/tc/140

DOI : $10.4000 /$ tc. 140

ISSN : 1952-420X

Éditeur

Éditions de l'EHESS

\section{Édition imprimée}

Date de publication : 1 avril 2004

Pagination : 175-177

ISSN : 0248-6016

\section{Référence électronique}

Alain Testart, «Réponse à François-René Picon, «Ce qu'esclave veut dire »», Techniques \& Culture [En ligne], 42 | 2004, mis en ligne le 06 novembre 2007, consulté le 29 septembre 2022. URL : http:// journals.openedition.org/tc/140 ; DOI : https://doi.org/10.4000/tc.140

Ce document a été généré automatiquement le 29 septembre 2022.

Tous droits réservés 


\title{
Réponse à François-René Picon, « Ce qu'esclave veut dire »
}

\author{
Alain Testart
}

1 Je ne voudrais pas chercher querelle à un collègue et un ami qui signe dans une des dernières livraisons de Techniques \& culture ${ }^{1}$ un compte rendu élogieux et fort honorable de mon livre sur l'esclavage. Toutefois, les trois ou quatre pages qu'il consacre aux Guajiros laissent à penser que les bases de données sur lesquelles je m'appuie (relatives à l'esclavage et aux prestations matrimoniales) ont été faites à la va-vite et contiennent des erreurs grossières. Je ne crois pas que ce soit le cas. Ces deux bases ont été réalisées en équipe; l'ensemble des documents relatifs à un peuple a été lu et analysé indépendamment par au moins deux membres de cette équipe; chaque fois qu'une décision de codage paraissait mal assurée, nous avons préféré mettre un point d'interrogation; enfin, les notes analytiques (en dehors de la photocopie des sources) couvrent plusieurs milliers de pages, qu'il était évidemment inconcevable de reproduire au sein d'un petit livre synthétique.

2 La controverse porte sur trois points. F.-R. Picon dit que je dis qu'il y a esclavage pour dettes chez les Guajiros, et ne consacre pas moins de deux pages à me réfuter. Mais je ne dis pas cela. Je dis même explicitement le contraire (Testart $2004: 185$ ), puisque, après avoir évoqué la mise en gage de la personne chez les Yurok, j'ajoute: "On retrouve peut-être cette institution chez les Guajiros (Gutiérrez de Pineda 1950 : 147 sq.; Bolinder 1957: $61 \mathrm{sq}$.): la dépendance des endettés y était très marquée mais les sources ne disent pas assez précisément si le travail remboursait la dette ou non. » Or, j'oppose très fortement gagé pour dette et esclave pour dettes (c'est même un des thèmes majeurs du livre). Au demeurant, comme -au moins dans certaines sources-il est question que le travail rembourse la dette, nous nous trouvons dans une situation encore plus éloignée de l'esclavage pour dettes (cette situation étant la troisième que j'oppose à cet esclavage pour dettes). Enfin, les sources étant à la fois anciennes et confuses, nous avons préféré mettre un point d'interrogation (ce que l'on voit sur la carte de l'esclavage pour dettes, p. 183) pour signaler le problème. 
3 L'esclavage pour dettes n'étant qu'un cas particulier de l'esclavage (une des sources, à côté d'autres), cela ne présume pas de savoir s'il y a ou non de l'esclavage chez les Guajiros (mais pour d'autres raisons, bien sûr). F.-R. Picon dit qu'il n'y en a pas. Mais tous les autres auteurs disent qu'il en y a, en particulier Gutiérrez de Pineda et Bolinder, qui constituent des références majeures en matière d'ethnohistoire sur les Guajiros. Tous deux parlent de guerres intestines au cours desquelles le groupe vaincu était exterminé, hormis quelques individus qui étaient retenus comme captifs; ces gens que l'on pouvait vendre, que l'on pouvait tuer à volonté, et qui étaient de surcroît hors parenté, c'est ce que j'appelle des «esclaves » puisqu'ils remplissent parfaitement nos critères de dépendance et d'exclusion. Et on ne voit pas bien les arguments qui permettent à F.-R. Picon d'affirmer qu'il n'y avait pas d'esclavage en Guajira. Il nous dit même que les données issues des HRAF «ne sont pas pour autant dépassées » (p. 160), et ces données sont essentiellement celles de Gutiérrez de Pineda et Bolinder qui consacrent à eux deux des dizaines de pages à l'esclavage : pourquoi F.-R. Picon n'en parle-t-il pas ? Il admet que "certains caciques guajiros étaient [...] accompagnés d'"esclaves" noirs». J'ai le regret de ne pas comprendre l'argument, et surtout pas pourquoi le qualificatif d'esclaves est entre guillemets s'agissant des Noirs : ne serait-ce pas de vrais esclaves? n'y aurait-il pas eu d'esclaves noirs en Amérique latine ? ou bien l'esclavage des Noirs est-il tellement banalisé dans ces contrées qu'il faut le tenir pour non significatif? Il y a longtemps qu'on a montré, au moins pour les Cherokee (il est vrai que nous sommes dans ce cas en Amérique du Nord), que les Amérindiens qui avaient des Noirs pour esclaves au XVII ${ }^{e}$ siècle. Ils avaient aussi des esclaves indiens, et ce, avant d'en avoir des noirs. Quant à l'argument du modèle lignager, et plus particulièrement nuer (pp. 162-63), il est particulièrement malvenu puisque la plupart (la totalité à quelques exceptions près) des sociétés lignagères africaines avaient des esclaves : c'est là une chose parfaitement documentée à l'heure actuelle et, au surplus, un des thèmes majeurs de mon livre. Et encore, sur les Nuer eux-mêmes, je leur consacre deux pages (Testart $2001: 27-28$ ), presque en ouverture du livre, au premier chapitre, pour affirmer qu'en dépit du silence d'Evans-Pritchard sur la question, on ne voit pas ce que pourrait être ces captifs dinka non encore adoptés, ces dépendants des Nuer qui vivaient avec eux et qui pouvaient être tués à volonté, si ce n'est des esclaves.

4 J'en viens au dernier point qui pourra être plus bref parce qu'il repose sur un pur contresens. F.-R. Picon me tance parce que, dit-il, et contrairement à ce que j'écris, il y a un retour du prix de la fiancée : un homme, après avoir payé le prix de la fiancée pour l'obtention de son épouse, touchera le prix de la fiancée pour la fille qu'il aura de cette épouse. C'est bien évident, et tout à fait général. La considération de cette donnée, d'ailleurs parfaitement bien connue et souvent notée dans les ethnographies (un homme qui a beaucoup de filles devient riche, et a donc fait un bon investissement), ne peut pas servir à différencier les formes de prestations matrimoniales. Et si j'avais employé le mot « retour » en ce sens, j'aurais été un parfait imbécile. Aussi n'est-ce pas en ce sens que j'ai employé ce mot, mais au sens de savoir si oui ou non le père de la fiancée, après avoir reçu le prix de la fiancée, retournait à son gendre une part significative de biens, sous forme de dot, donc. Cela est expliqué de façon brève, mais claire, je crois, à la page 186 de mon livre sur l'esclavage, mais l'a été dans maintes autres publications sur la question (l'article cosigné par moi-même et al, faisant référence, $2002: 173-4)$. Chaque fois que la part que fournit le père en retour du prix de la fiancée ne dépasse pas $50 \%$ du montant du prix de la fiancée, nous avons parlé de prix de la fiancée "sans retour ", c'est-à-dire un prix de la fiancée que l'on ne peut pas 
considérer comme une combinaison prix de la fiancée-dot : et, bien qu'il soit toujours difficile d'estimer la valeur des biens dans ce type de société, aucune des ethnographies, y compris celles de F.-R. Picon que nous avons prises en compte, ne montre qu'il y aurait un retour important de la part du père chez les Guajiros.

\section{BIBLIOGRAPHIE}

Bolinder, G.

1957. Indians on Horseback. Londres : Dennis Dolson.

Gutiérrez de Pineda, V.

1950. Organización social en la Guajira. Bogotá [trad. HRAF].

Testart, Alain

2001. L'esclave, la dette et le pouvoir : Études de sociologie comparative. Paris : Errance.

Testart, Alain, Nicolas Govoroff \& Valérie Lécrivain

2002. « Les prestations matrimoniales », L'Homme 161 : 165-196.

\section{NOTES}

1. 41, 2003 : 153-167. F.-R. Picon, « Ce qu'esclave veut dire », Chroniques et commentaires, à propos du livre d'Alain Testart: L'esclave, la dette et le pouvoir.

\section{AUTEUR}

\section{ALAIN TESTART}

LAS, CNRS 